\title{
A case of myeloperoxidase anti-neutrophil cytoplasmic antibody (MPO-ANCA)-associated glomerulonephritis and concurrent membranous nephropathy
}

Michiko Shimada1", Takeshi Fujita', Norio Nakamura², Ikuyo Narita' ${ }^{1}$, Yuko Shimaya', Reiichi Murakami', Hideaki Yamabe ${ }^{1}$, Hiroshi Osawa ${ }^{1}$ and Ken Okumura ${ }^{1}$

\begin{abstract}
Background: Myeloperoxidase anti-neutrophil cytoplasmic antibody-associated glomerulonephritis (MPO-ANCA-GN) and concurrent membranous nephropathy (MN) are very rare combination. Their causal relationship has been suggested, but not determined.

Case presentation: A 73-years-old male with 5-year history of proteinuria underwent an operation for his sigmoid colon cancer. Seven months later, he was referred to a nephrology division due to an exacerbating renal function and hypoalbuminemia. Laboratory examination revealed positive MPO-ANCA in the serum. A renal biopsy revealed a necrotizing extracapillary proliferative glomerulonephritis with crescents, demonstrating MPO-ANCA-GN. Whereas, immunofluorescent staining documented granular deposition of immumoglobulin (lg) G and C3 along the capillary wall and electron microscopy showed subepithelial deposits in the glomerular basement membrane demonstrating MN. Immunofluorescent staining of $\lg G$ subclass showed positive $\lg G 1, \lg G 2$, negative $\lg G 3$ and weak positive $\lg G 4$ suggested the possibility of malignancy-associated MN.
\end{abstract}

Conclusion: Combination of MPO-ANCA-GN and MN are rare. Although the causal relationship has been suggested in some cases, we should consider all the possibilities including idiopathic MN and secondary MN associated with malignancy, drug use or infection.

Keywords: MPO-ANCA, Crescentic glomerulonephritis, Membranous nephropathy

\section{Background}

Anti-neutrophil cytoplasmic antibody-associated glomerulonephritis (ANCA-GN) is usually characterized by necrotizing and crescentic glomerulonephritis without the deposition of immunoglobulin and complement, therefore, it is called pauci immune type. Whereas, immunoglobulin depositions have been sometimes observed [1] and very rare cases of membranous nephropathy (MN) and the concurrent ANCA-GN and have been suggested [2-6]. The precise mechanism of the combination of these two etiologies is not clear due to the limited numbers of the

\footnotetext{
*Correspondence: mshimada@cc.hirosaki-u.ac.jp

${ }^{1}$ Graduate School of Medicine, Division of Cardiology, Respiratory Medicine and Nephrology, Hirosaki University, 5 Zaifu-cho, Hirosaki 036-8562, Japan Full list of author information is available at the end of the article
}

patients. Here we describe a case of myeloperoxidase (MPO)-ANCA-GN complicated with MN developed in 73-years-old male.

\section{Case presentation}

A 73-years-old male underwent a curative operation for a well-differentiated adenocarcinoma of the sigmoid colon. The tumor was (T2) with no lymphatic invasion (N0) and no distant metastasis (M0). Seven months later, he was referred to a nephrology division and admitted due to an exacerbating renal function and hypoalbuminemia without any clinical symptoms other than edema in the lower extremities. He had been diagnosed with hypertension, vasospastic angina and gastric ulcer for 11 years and treated with olmesartan medoxomil, nicorandil and roxatidine 

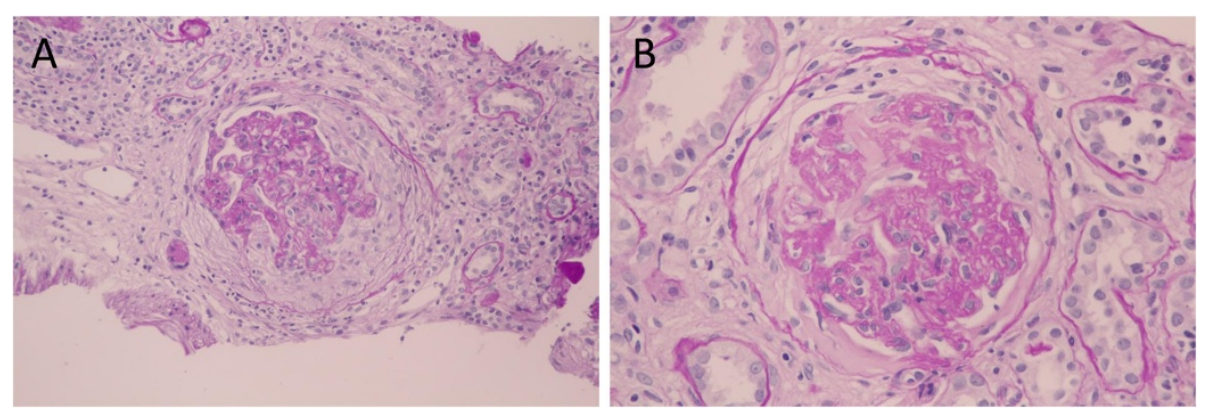

Figure 1 Light microscopic findings. Periodic acid-Schiff's staining reveal a necrotizing extracapillary proliferative glomerulonephritis with crescent and many infiltrated mononuclear cells in the tubulointerstitium (A: original magnification $\times 200$ ). A glomerulus with fibrocellular crescent and thickened glomerular basement membrane $(G B M)(B$ : original magnification $\times 400)$. A glomerulus with fibrocellular crecent and thickened glomerular basement membrane (GBM) (Periodic acid-Schiff's stain, original magnification $\times 400)$.

acetate. Proteinuria had been detected for 5 years $(++\sim+$ $++)$. At the time of the pre-operative examinations, there was no specific symptoms. Serum creatinine was stable $(1.2 \mathrm{mg} / \mathrm{dl}), \mathrm{C}$-reactive protein $(\mathrm{CRP})$ was negative, proteinuria $(++)$ and microhematuria was absent. Laboratory values at the time of referral are as follows: hemoglobin $10.1 \mathrm{~g} / \mathrm{dL}$, total protein $6.7 \mathrm{~g} / \mathrm{dL}$, albumin $3.1 \mathrm{~g} / \mathrm{dL}$, lactate dehydrogenase $208 \mathrm{U} / \mathrm{L}$, blood urea nitrogen $59 \mathrm{mg} / \mathrm{dL}$, creatinine $5.4 \mathrm{mg} / \mathrm{dL}$, CRP $0.88 \mathrm{mg} / \mathrm{dL}$, carcinoembryonic antigen (CEA) $4.1 \mathrm{ng} / \mathrm{ml}$ (negative), carbohydrate antigen (CA) $19-9<1 \mathrm{U} / \mathrm{ml}$ (negative), MPO-ANCA $>640 \mathrm{EU}$ (positive), PR3-ANCA tested by ELISA $<10$ EU (negative), anit-glomerular besement membrane (GBM) antibody $<10 \mathrm{EU}$ (negative), anti nuclear antibody $\times 40$ (normal limit < ×40), C3 97mg/dl, C4 59mg/dl. Urinalysis showed hematuria $(+++)$ : sediment red blood cells 40 /high power field and proteinuria $2.3 \mathrm{~g} /$ day. Chest CT scan revealed no specific findings. A renal biopsy was performed and documented a necrotizing extracapillary proliferative glomerulonephritis. There were 11 glomeruli, 5 with global sclerosis, 8 with mostly segmental crescents (5 fibro-cellular, 3 cellular) (Figure 1A, B). Routine immunofluorescent analysis revealed granular immumoglobulin $\mathrm{G}$ (IgG) and C3 deposition along the glomerular capillary wall (Figure 2A, B). IgA, IgM and fibrionogen were negative. Glomerular IgG subclass determined by immunofluorescent analysis showed positive IgG1 and IgG2, negative IgG3 and weak positive IgG4 deposition (Figure 3 A-D). Electron microscopy revealed electron-dense deposits in the subepithelial area of the GBM suggesting stage $3 \mathrm{MN}$ (Figure 4).

3 week pulse therapy with methylprednisolone (500 $\mathrm{mg}$ for 3 days/week) followed by oral prednisolone (30 mg/day) decreased the levels of serum MPOANCA to normal range. Microhematuria was disappeared and CRP became negative, however, the levels of proteinuria and renal function did not improve. In a month, the antigenemia of the cytomegalovirus became positive without any clinical symptoms and was treated with valganciclovir. Therefore, prednisolone was tapered without adding immunosuppressants. Hemodialysis was initiated 6 months after the diagnosis of MPO-ANCA-GN. There was no recurrence of colon cancer and MPO-ANCA remained negative during the follow-up.
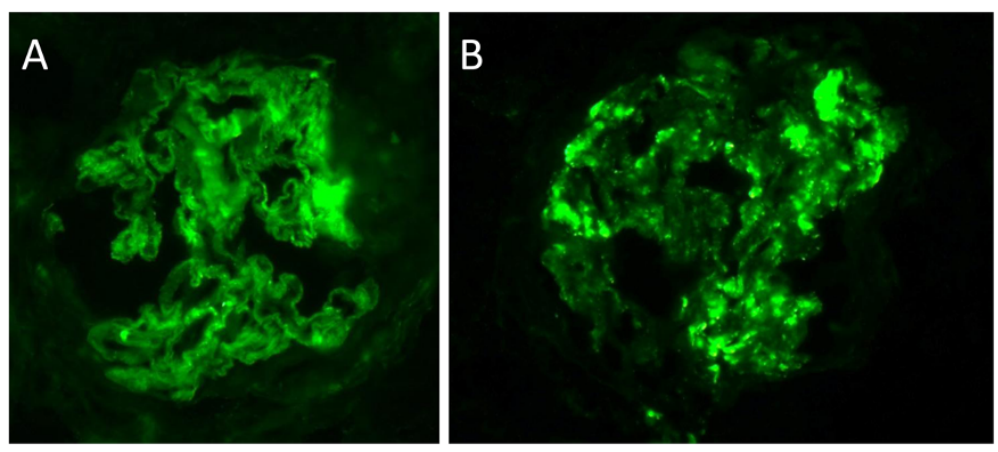

Figure 2 Immunofluorescent staining for $\lg G(A)$ and $C 3(B)$ reveals granular deposition in the glomerular capillary wall (original magnification $\times 400$ ). 

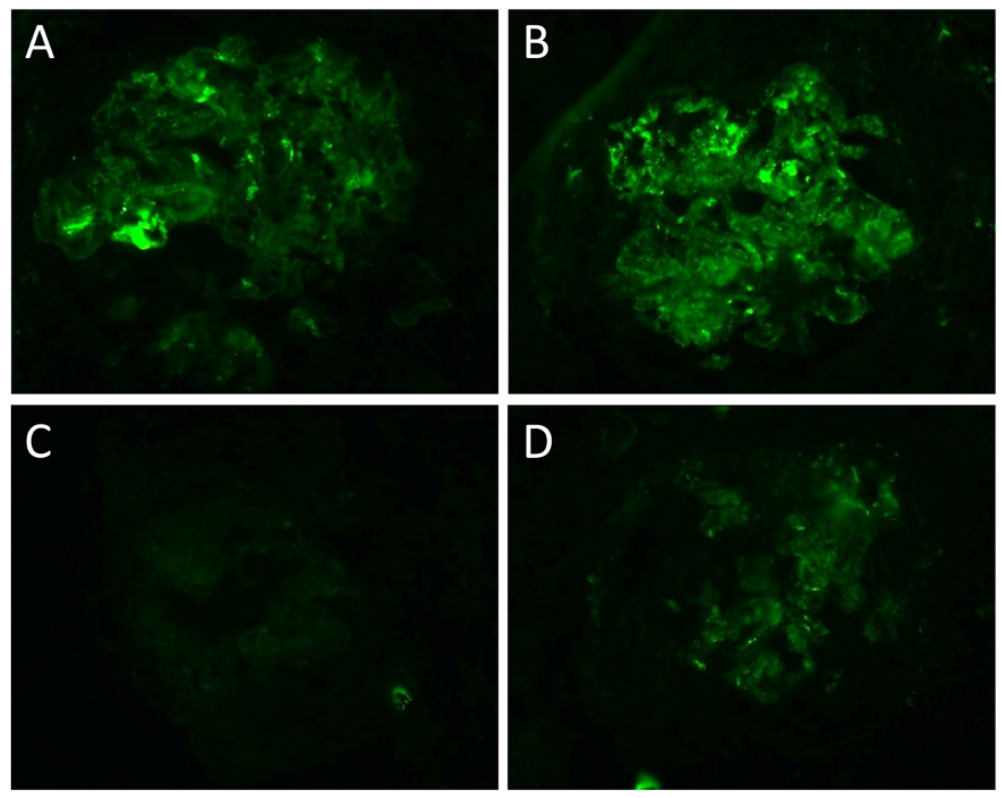

Figure 3 Immunofluorescent staining for IgG subclass. $\lg G 1(\mathbf{A})$ and $\lg G 2(B)$ reveals granular deposition in the glomerular capillary wall. $\lg \mathrm{G} 3(\mathbf{C})$ was negative and $\lg \mathrm{G} 4(\mathbf{D})$ was weak positive (original magnification $\times 400$ ).

\section{Discussion}

$\mathrm{MN}$ and concurrent MPO-ANCA-GN are very rare combination. It has been a question whether this is just a coincidence or there is a causal relationship. Recently, Hamamura et al. showed that MPO-ANCA-GN may cause MN-like lesions by demonstrating partial colocalization of MPO and IgG within the electron dense deposits [7]. There are also several characteristics observed in concurrent MN and ANCA-GN. Granular deposition of IgG and C3 along the GBM is similar, but IgG subclass deposition is different from predominant IgG4 pattern in idiopathic-MN and IgG1 and IgG4 depesition is reported in several cases [6,7]. This is compatible with the fact that the serum subclass of MPOANCA consisted mainly of IgG1 and IgG4 [8]. Besides, the electron microscopy findings show irregular dense deposits different from the global pattern of the idiopathic-MN. Due to the small numbers of the cases, further investigation is necessary for further understanding of the relationship between these two etiologies. In most cases, these two etiologies found concurrently, therefore, causal relationship is quite probable $[2,5,7]$. On the other hand, this may not explain all the cases, especially when $\mathrm{MN}$ and ANCA-GN diagnosed at different time course. In some cases, MPO-ANCA-GN was found during the remission of the $\mathrm{MN}[3,5]$. These cases may be truly coincidental occurrence of the idiopathic-MN and MPO-ANCA-GN. Additionally, other causes such as malignancy-associated, infection-associated and druginduced MN should be considered as well. In our case,
MN and MPO-ANCA-GN were diagnosed simultaneously, but the long duration of the proteinuria and the colon cancer suggested the possibility of malignancy associated-MN which presumably preceded MPO-ANCA -GN at least to some extent. Electron microscopy findings also showed dense deposits of stage 3, relatively old lesion of MN. Additionally, predominant IgG1 and IgG2 deposition also suggested that $\mathrm{MN}$ in our case was secondary MN due to malignancy [9]. However, since we could not see the obvious improvement of proteinuria after the surgery of the colon cancer, we could not definitively diagnose that this case was malignancy associated-MN. Thus,

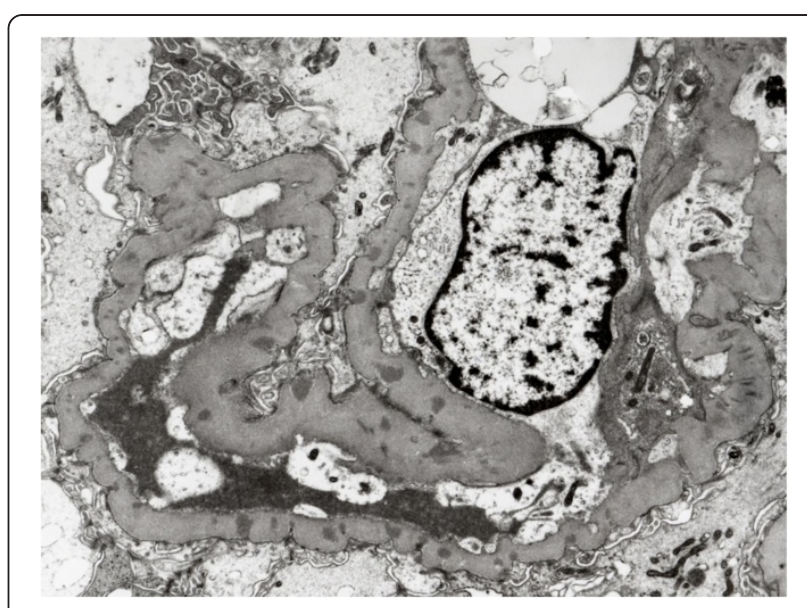

Figure 4 Electron microscopy shows electron dense deposits in subepithelial area of GBM (original magnification $\times 5000$ ). 
concurrent MN and MPO-ANCA-GN possibly include different etiologies. Precise observation of these cases, especially the onset of the diseases, the analysis of IgG subclass in the glomerular deposition and the examination for the existence of the anti M-type phospholipase A2 receptor antibody which is demonstrated as the cause of idiopatic-MN also would help further understanding of these cases [10].

\section{Conclusions}

Causal relationship has been suggested in the combination of MPO-ANCA-GN and MN in some cases, however, we should consider all the possibilities including malignancy, drug or infection associated $\mathrm{MN}$ as well as idiopathic MN. Besides, the analysis of the IgG subclass in the glomerular deposition may be of help.

\section{Consent}

Written informed consent was obtained from the patient for publication of the study in the BMC Nephrology.

\section{Competing interests}

The authors declare that they have no competing interests.

\section{Authors' contributions}

MS wrote the manuscript and was a treating physician for the patient. TF, $\mathrm{NN}, \mathrm{IN}, \mathrm{YS}$, and RM were also treating physicians for the patient. $\mathrm{YH}, \mathrm{HO}, \mathrm{KO}$ performed the literature search and helped to write the manuscript. All authors read and approved the final manuscript.

\section{Author details}

${ }^{1}$ Graduate School of Medicine, Division of Cardiology, Respiratory Medicine and Nephrology, Hirosaki University, 5 Zaifu-cho, Hirosaki 036-8562, Japan. ${ }^{2}$ Graduate School of Medicine, Community Medicine, Hirosaki University,

5 Zaifu-cho, Hirosaki 036-8562, Japan.

Received: 13 August 2012 Accepted: 12 March 2013

Published: 28 March 2013

\section{References}

1. Haas M, Eustace JA: Immune complex deposits in ANCA-associated crescentic glomerulonephritis: a study of 126 cases. Kidney Int 2004, 65(6):2145-2152.

2. Nasr SH, Said SM, Valeri AM, Stokes MB, Masani NN, D'Agati VD, Markowitz GS: Membranous glomerulonephritis with ANCA-associated necrotizing and crescentic glomerulonephritis. Clin J Am Soc Nephrol 2009, 4(2):299-308.

3. Kanahara K, Yorioka N, Nakamura C, Kyuden Y, Ogata S, Taguchi T, Yamakido M: Myeloperoxidase-antineutrophil cytoplasmic antibodyassociated glomerulonephritis with membranous nephropathy in remission. Intern Med 1997, 36(11):841-846.

4. Dwyer KM, Agar JW, Hill PA, Murphy BF: Membranous nephropathy and anti-neutrophil cytoplasmic antibody-associated glomerulonephritis: a report of 2 cases. Clin Nephrol 2001, 56(5):394-397.

5. Tse WY, Howie AJ, Adu D, Savage CO, Richards NT, Wheeler DC, Michael J: Association of vasculitic glomerulonephritis with membranous nephropathy: a report of 10 cases. Nephrol Dial Transplant 1997, 12(5):1017-1027.

6. Suwabe T, Ubara Y, Tagami T, Sawa N, Hoshino J, Katori H, Takemoto F, Hara S, Aita K, Hara S, et al: Membranous glomerulopathy induced by myeloperoxidase-anti-neutrophil cytoplasmic antibody-related crescentic glomerulonephritis. Intern Med 2005, 44(8):853-858.

7. Hanamura K, Tojo A, Kinugasa S, Asaba K, Onozato ML, Uozaki H, Fukayama M, Fujita T: Detection of myeloperoxidase in membranous nephropathylike deposits in patients with anti-neutrophil cytoplasmic antibodyassociated glomerulonephritis. Hum Pathol 2011, 42(5):649-658.

8. Segelmark M, Wieslander J: IgG subclasses of antineutrophil cytoplasm autoantibodies (ANCA). Nephrol Dial Transplant 1993, 8(8):696-702.
9. Ohtani H, Wakui H, Komatsuda A, Okuyama S, Masai R, Maki N, Kigawa A, Sawada K, Imai H: Distribution of glomerular lgG subclass deposits in malignancy-associated membranous nephropathy. Nephrol Dial Transplant 2004, 19(3):574-579.

10. Beck LH Jr, Bonegio RG, Lambeau G, Beck DM, Powell DW, Cummins TD, Klein JB, Salant DJ: M-type phospholipase A2 receptor as target antigen in idiopathic membranous nephropathy. New Engl J Med 2009, 361(1):11-21.

doi:10.1186/1471-2369-14-73

Cite this article as: Shimada et al:: A case of myeloperoxidase antineutrophil cytoplasmic antibody (MPO-ANCA)-associated glomerulonephritis and concurrent membranous nephropathy. BMC Nephrology 2013 14:73.

\section{Submit your next manuscript to BioMed Central and take full advantage of:}

- Convenient online submission

- Thorough peer review

- No space constraints or color figure charges

- Immediate publication on acceptance

- Inclusion in PubMed, CAS, Scopus and Google Scholar

- Research which is freely available for redistribution 